TUW-00-18

\title{
Renormalizability of the open string sigma model and emergence of $D$-branes
}

\author{
W. Kummer ${ }^{1 *}$ and D.V. Vassilevich ${ }^{2 \dagger}$ 用 \\ ${ }^{1}$ Institut für Theoretische Physik, Technische Universität Wien \\ Wiedner Hauptstr. 8-10, A-1040 Wien \\ Austria \\ ${ }^{2}$ Institut für Theoretische Physik, Universität Leipzig, \\ Augustusplatz 10, D-04109 Leipzig, \\ Germany
}

\begin{abstract}
Rederiving the one-loop divergences for the most general coupling of the open string sigma model by the heat kernel technique, we distinguish the classical background field from the mean field of the effective action. The latter is arbitrary, i.e. does not fulfil the boundary conditions. As a consequence a new divergent counter term strongly suggests the introduction of another external one-form field (beside the usual gauge field), coupled to the normal derivative at the boundary. Actually such a field has been proposed in the literature for different reasons, but its full impact never seems to have thoroughly investigated before. The beta function for the resulting renormalizable model is calculated and the consequences are discussed, including the ones for the Born-Infeld action. The most exciting property of the new coupling is that it enters the coefficient in front of the normal derivative in Neumann boundary conditions. For certain values of the background fields this coefficient vanishes, leading to Dirichlet boundary conditions. This provides a natural mechanism for the emergence of D-branes.
\end{abstract}

PACS: 11.25.-w, keywords: open strings, renormalization, heat kernel

*e-mail: wkummer@tph.tuwien.ac.at

$\dagger$ e-mail: Dmitri.Vassilevich@itp.uni-leipzig.de

${ }^{\ddagger}$ On leave from Department of Theoretical Physics, St. Petersburg University, 198904 St. Petersburg, Russia 


\section{Introduction}

An essential ingredient for the proper formulation of systems consisting of strings and (D-) branes is the Born-Infeld (BI) action which originally had been introduced in string theory by Fradkin and Tseytlin [1,2]. Other pioneering works [3, [4] re-derived it from the condition that the beta-function for the string vanishes when the string is embedded in an external gauge field $A_{\mu}(X)$, where $X^{\mu}$ is the target space coordinate. Previously the beta-function method was successfully applied to closed strings [5]. From the extensive literature on the application of this action in brane theory and its vast number of consequences we only refer to a review [6].

The bulk contribution of the sigma model from the usual string arguments also contains the two-form gauge field $B$ and two scalar fields $(\Phi, T)$ one of which $(\Phi)$ is coupled to the world volume Ricci scalar on the (compact) string manifold $\mathcal{M}_{2}=\mathcal{M}$. In the presence of a boundary $\partial \mathcal{M}$, there beside the oneform gauge field $A$ two similar scalar fields $(\hat{\Phi}, \hat{T})$ may appear, of which $\hat{\Phi}$ is coupled to extrinsic curvature. It has been suggested [7-11] (e.g. from arguments like duality) to introduce also another one-form field $V_{\mu}$ coupled to the normal derivative of $X^{\mu}$ on $\partial \mathcal{M}$, however we are not aware of a systematic treatment of the problems including all consequences of that extension. It will be the main issue of our present work to show that renormalizability at the boundary strongly suggests the inclusion of $V$. As we explain below, this means an extension of the approach 44 to achieve full renormalization of the theory. At the same time we discover a natural mechanism for the emergence of $D$-branes.

We have found the heat kernel technique [12] especially convenient for a treatment of the one-loop quantum effects, because also for problems with nontrivial boundary many mathematical rigorous results are available which may be used in the present context. Our argument relies upon the fact that for the one-particle irreducible (1-p-i) loop integral the external lines refer to the "mean field" $\bar{X}$, which is arbitrary, and, in particular, does not fulfil the boundary conditions.

For this reason in Sect. 2 the relation of $\bar{X}$ to the background field, appearing in the path integral of the generating functional of Green functions is clarified. Instead of doing the Legendre transformation in the path integral - a procedure which may introduce certain difficulties in the presence of boundaries - we show how to construct directly the functional which generates the one-loop 1-p-i diagrams. At this order, this functional is equivalent to just one diagram with the propagator modified by all possible vertex insertions. The sketch of a more formal derivation of the same result where the Legendre transform is used, for a general action with boundary is presented in Appendix A.

In Sect. 3 we return to the determination of the divergent contribution of the one-loop corrections for the usual sigma-model with boundary (without second 1form field $V$ ), using the powerful heat kernel technique. Some mathematic issues as, for example, strong ellipticity of the boundary value problem are clarified. We 
rederive the divergent part of the one-loop effective action and, as a by-product, we generalize and extend the results existing in the literature [4, 17, 13]. On the basis of the analysis in the previous Section we suggest an extension of the method of Callan and coworkers [4 which amounts to the inclusion of the $V$-coupling at the boundary.

In Section 4 we investigate the consequences of introducing the vector field $V_{\mu}$, coupled to $\partial_{N} X^{\mu}$ in the action, right from the start. For simplicity we drop in that Section all dimensional couplings. We analyse the corresponding boundary value problem and define modified boundary conditions. An important point is to achieve hermiticity of the relevant operator. The $\beta$-functions are explicitly calculated to the leading order of $V$. We show that the expansion in the $V$ field generates derivative corrections to the Born-Infeld action with respect to $B_{\mu \nu}$. If $V$ is no longer small we observe a mechanism for spontaneous $D$-brane creation, which seems to be the most remarkable property of the proposed model. Generically, a $D$-brane is separated by a non-perturbative region populated by tachyonic modes.

\section{Effective action in the presence of boundaries}

The basis of the computation of quantum corrections, including renormalization and beta-functions, are 1-p-i vertex functions. Generally these vertices are obtained by functional differentiation with respect to an (arbitrary) "mean field" $\bar{X}$ from the effective action $W(\bar{X})$. In order to see that this mean field in $W$ is different from a classical background field we consider as an illustration a theory described by the classical action in Euclidean in $d=2$

$$
S=\int_{\mathcal{M}}\left(-(\partial X)^{2}+w(X)\right)+\int_{\partial \mathcal{M}}\left(-V(X) \partial_{N} X+\hat{w}(X)\right),
$$

where $w(X)$ and $\hat{w}(X)$ are some "potential" terms which may contain linear first derivatives (in the case of $\hat{w}$ these can be tangential derivatives to the boundary $\partial \mathcal{M}$ only). $\partial_{N}$ is the partial derivative with respect to the inward pointing unit vector on $\partial \mathcal{M}$. In anticipation of the situation for the string, to be discussed below, we also allow for a boundary term $V \partial_{N} X$ with normal derivative. Our strategy is to derive the effective action as the inverse of the full one-loop propagator in the presence of a boundary.

The action (11) is split into two parts:

$$
S=S_{\text {prop }}+S_{\text {int }}, \quad S_{\text {prop }}=-\int_{\mathcal{M}}(\partial X)^{2} .
$$

$S_{\text {prop }}$ will be used to define propagator, while $S_{\text {int }}$ determines the vertices. Usually

the quadratic part of $w$ is included in $S_{\text {prop }}$. However, since we are going to sum 
anyway all possible insertions we are allowed to include the quadratic terms in $S_{\text {int }}$. The propagator following from $S_{\text {prop }}$ above is

$$
G_{0}(x, y)=\left(-\partial^{2}\right)_{x, y}
$$

which is subject to boundary conditions defined by the boundary part of $S_{\text {prop }}$. These can be either of Dirichlet

$$
\left.G_{0}(x, y)\right|_{x \in \partial \mathcal{M}}=0
$$

or of Neumann type

$$
\left.\partial_{N}^{x} G_{0}(x, y)\right|_{x \in \partial \mathcal{M}}=0 .
$$

Theories described by these two types of the boundary conditions are different already at the classical level. Here we consider Neumann conditions (5) only, because their specific feature will be essential for our work below.

For our purposes it will be enough to treat 1-p-i Feynman diagrams with $n$ external lines. In coordinate space any such diagram is given by an $n$-point function $\mathcal{W}_{(n)}\left(x_{1}, \ldots, x_{n}\right)$. The renormalization problem refers to amputated diagrams. This means that the external lines just label arguments in the vertices. Thus to obtain the one-loop effective action the mean field $\bar{X}\left(x_{i}\right)$ may be simply attached at the vertices. Summing all such diagrams

$$
W(\bar{X})=\sum_{(n)} \int d x_{1} \ldots \int d x_{n} \mathcal{W}_{(n)}\left(x_{1}, \ldots, x_{n}\right) \bar{X}\left(x_{1}\right) \ldots \bar{X}\left(x_{n}\right)
$$

the effective action $W$ is obtained to one-loop. Clearly by functional differentiation with respect to the mean field $\bar{X}$ the individual 1-p-i diagrams are recovered.

The correct combinatorial coefficients of the vertex insertions in the propagator can be obtained by taking, as a first step, $S_{\text {int }}(\bar{X}+\xi)$, keeping only the second order terms in $\xi$ :

$$
S_{\text {int }}^{(2)}=\frac{1}{2} \int_{\mathcal{M}} \xi w^{\prime \prime}(\bar{X}) \xi+\int_{\partial \mathcal{M}}\left(-V^{\prime}(\bar{X}) \xi \partial_{N} \xi-\frac{1}{2} \xi V^{\prime \prime}(\bar{X}) \xi \partial_{N} \bar{X}+\frac{1}{2} \xi \hat{w}^{\prime \prime}(\bar{X}) \xi\right)
$$

Then the propagator $G_{1}(x, y)$ generated by all insertions of boundary vertices in $G_{0}(x, y)$ becomes

$$
\begin{aligned}
& G_{1}(x, y)=\sum_{k=0}^{\infty} \int_{\partial \mathcal{M}} d x_{1} \ldots \int_{\partial \mathcal{M}} d x_{k}(-1)^{k} G_{0}\left(x, x_{1}\right) U\left(x_{1}\right) G\left(x_{1}, x_{2}\right) \ldots U\left(x_{k}\right) G_{0}\left(x_{k}, y\right) \\
& U(x)=-\overleftarrow{\partial}_{N} V^{\prime}(\bar{X})-V^{\prime}(\bar{X}) \vec{\partial}_{N}-\frac{1}{2} V^{\prime \prime}(\bar{X})\left(\partial_{N} \bar{X}\right)+\frac{1}{2} \hat{w}^{\prime \prime}(\bar{X}) .
\end{aligned}
$$

It should be noted that the normal derivative appears twice in the boundary vertex operator $U$. This happens because on the boundary $\xi$ and $\partial_{N} \xi$ should 
be considered as independent. All correlation functions of the field $\xi$ should follow by variation of the path integral containing $\int_{\mathcal{M}}(j \xi)$ with respect to the source $j$. If one needs correlators containing $\xi(x)$, the source $j$ should also include a term behaving like a $\delta$-function at the boundary. To obtain derivatives of $\xi$ that part of the source should contain also a derivative of the $\delta$-function and an integration by parts should be performed. This does not work, however, if one needs normal derivatives of $\xi$ on the boundary. Then that integration by parts of terms like $\int_{\mathcal{M}} \xi \partial_{N} \delta(\partial \mathcal{M})$ would lead to a meaningless expression containing a factor $(\delta(\partial \mathcal{M}))^{2}$ in the integral (with one delta function coming from the integrand, and the other describing the surface contribution). To overcome this difficulty we introduce an independent field $\chi=\partial_{N} \xi$ on the boundary with the propagators $G_{0}^{\chi \xi}(x, y)=\partial_{N}^{x} G_{0}(x, y)$ and $G_{0}^{\chi \chi}(x, y)=\partial_{N}^{x} \partial_{N}^{y} G_{0}(x, y)$. Note, that one cannot put this propagator to zero if it appears under an integral over $x$ because of the singularity in $G_{0}$ for coinciding arguments. This leads to the effective doubling of the normal derivative term in $U$ in (8) because one should take into account both terms: ... $G_{0}^{\ldots \chi}\left(x_{n-1}, x_{n}\right)\left(-V^{\prime}\right) G_{0}^{\xi \ldots}\left(x_{n}, x_{n+1}\right) \ldots$ and $\ldots G_{0}^{\cdots \xi}\left(x_{n-1}, x_{n}\right)\left(-V^{\prime}\right) G_{0}^{\chi \ldots}\left(x_{n}, x_{n+1}\right) \ldots$

For the next step we note that $G_{1}(x, y)$ evidently satisfies the Dyson equation:

$$
G_{1}(x, y)=G_{0}(x, y)-\int_{\partial \mathcal{M}} d z G_{0}(x, z) U(z) G_{1}(z, y)
$$

In order to find out whether the correct boundary conditions are satisfied by $G_{1}(x, y)$ we let $\partial_{N}^{x}$ act on both sides of the equation (9) and put $x$ onto the boundary:

$$
\left.\partial_{N}^{x} G_{1}(x, y)\right|_{x \in \partial \mathcal{M}}=-\lim _{x \rightarrow \partial \mathcal{M}} \int_{\partial \mathcal{M}} d z \partial_{N}^{x} G_{0}(x, z) U(z) G_{1}(z, y)
$$

To calculate the right hand side of (10) we restrict ourselves to the case of the half-plane, $\mathcal{M}=\mathbf{R} \times \mathbf{R}_{+}$. As we will see below, only the leading short-distance singularity of the propagator $G_{0}(x, z)$ contributes to (10). At short distances all two dimensional manifolds with boundary, as far as the leading singularity is concerned, can be treated as a half-plane. This restriction allows to take the $2 \mathrm{~d}$ propagator $G_{0}$ simply as the one with a "mirror charge"

$$
G_{0}(x, z)=-\frac{1}{4 \pi}\left(\ln |x-z|^{2}+\ln \left|x-z^{*}\right|^{2}\right)
$$

where $z^{*}=\left(z^{1},-z^{2}\right)$. For symmetry reasons the contribution to (10) of the first term in $U$ of (8) vanishes. For the rest of $U$ we have

$$
\begin{aligned}
\left.\partial_{N}^{x} G_{1}(x, y)\right|_{x \in \partial \mathcal{M}}= & \frac{1}{4 \pi} \lim _{x^{2} \rightarrow+0} \int_{\partial \mathcal{M}} d z \frac{4 x^{2}}{\left(x^{2}\right)^{2}+\left(x^{1}-z^{1}\right)^{2}} \times \\
& \times\left(-V^{\prime}(\bar{X}) \vec{\partial}_{N}^{z}-\frac{1}{2} V^{\prime \prime}(\bar{X})\left(\partial_{N} \bar{X}\right)+\frac{1}{2} \hat{w}^{\prime \prime}(\bar{X})\right) G(z, y) \\
= & \left.\left(-V^{\prime}(\bar{X}) \vec{\partial}_{N}^{x}-\frac{1}{2} V^{\prime \prime}(\bar{X})\left(\partial_{N} \bar{X}\right)+\frac{1}{2} \hat{w}^{\prime \prime}(\bar{X})\right) G(x, y)\right|_{x \in \partial \mathcal{M}}
\end{aligned}
$$


where the identity

$$
\lim _{\epsilon \rightarrow 0} \frac{\epsilon}{\epsilon^{2}+z^{2}}=\pi \delta(z)
$$

has been used. It is straightforward to check that also

$$
-\partial^{2} G_{1}(x, y)=\delta(x-y) . \quad x, y \notin \partial \mathcal{M}
$$

Thus $G_{1}$ is in agreement with the boundary term in (7) and it represents a proper solution of $-\partial^{2}$. Finally we sum all the bulk insertions of (7) to the propagator $G_{1}(x, y)$. Clearly, the full propagator satisfies

$$
G(x, y)=G_{1}(x, y)-\int_{\mathcal{M}} d z G_{1}(x, z) \frac{1}{2} w^{\prime \prime}(\bar{X}(z)) G(z, y) .
$$

Since $w^{\prime \prime}(\bar{X}(z))$ is smooth on $\partial \mathcal{M}$ the boundary conditions remain valid for $G(x, y)$ as well as for $G_{1}(x, y)$. The bulk equation (14) is modified by the term proportional to $w^{\prime \prime}$

$$
\begin{aligned}
\partial_{x}^{2} G(x, y) & =-\delta(x, y)-\int_{\mathcal{M}} d z \partial_{x}^{2} G_{1}(x, z) \frac{1}{2} w^{\prime \prime}(\bar{X}(z)) G(z, y) \\
& =-\delta(x, y)+\frac{1}{2} w^{\prime \prime}(\bar{X}(x)) G(x, y)
\end{aligned}
$$

for $x, y \notin \partial \mathcal{M}$ so that $G$ contains the "mass" term in the proper way.

Thus we have proved that, after summing all insertions in the propagator $G_{0}(x, y)$ which are relevant for calculation of the one-loop effective action, one arrives at a propagator

$$
G(x, y)=\left(-\partial^{2}+\frac{1}{2} w^{\prime \prime}(\bar{X})\right)_{x, y}^{-1}
$$

defined on the space of fields satisfying generalized Neumann boundary condition

$$
\left.\left(-\partial_{N}-V^{\prime}(\bar{X}) \partial_{N}-\frac{1}{2} V^{\prime \prime}(\bar{X})\left(\partial_{N} \bar{X}\right)+\frac{1}{2} \hat{w}^{\prime \prime}(\bar{X})\right) \xi\right|_{\partial \mathcal{M}}=0 .
$$

¿From this we can conclude that the effective action becomes

$$
W(\bar{X})=\frac{1}{2} \ln \operatorname{det}\left(-\partial^{2}+\frac{1}{2} w^{\prime \prime}(\bar{X})\right),
$$

the "naive" result one would expect based upon experience for manifolds without boundaries.

Though our calculations were valid, strictly speaking, only for flat geometries, the generalization to curved manifolds with curved boundaries is straightforward, but technically involved. Another subtle point is the symmetry of the propagator 
$G(x, y)$ which is equivalent to the hermiticity of the operator in (16) when (18) holds. This problem will be discussed in sec. 4.2 .

In a suitable regularization the divergent part $W_{\text {div }}$ of $W(\bar{X})$ defines the counterterms which should introduced in the theory for renormalization. Any local invariant (bulk or boundary) appearing in $W_{\text {div }}$ corresponds to certain divergent diagrams just by definition of the effective action. It is clear therefore that we are not allowed to impose any restrictions, as e.g. boundary conditions, on the mean field $\bar{X}$. If $W(\bar{X})$ as a function of some restricted $\bar{X}$ is made finite, we cannot guarantee that the full theory will become finite as well. Of course, if we contract the mean field with external lines of a digram, the contributions proportional to the boundary operator will vanish. Therefore, to make the theory finite at one loop it is enough to remove divergences which are not annihilated by the boundary operator (as done in $\|$ ). However, if the mean field represents internal lines of a diagram, there could appear problems with renormalizability due to interference of singularities. It is not clear, to which order of perturbation theory a renormalization scheme with boundary conditions on the mean field could be extended (in certain models such an extension has been achieved up to two loops 14]).

The necessity to introduce independent counterterms was recognized long ago by Symanzik [15]. Also related work [16] should be mentioned where $\beta$-functions with Dirichlet boundary conditions were considered. Although in that paper the boundary value of the mean field had been fixed at some intermediate steps, in the calculations of the $\beta$-functions it was a free parameter generating all counterterms.

In the analysis of the renormalization of the bosonic sigma model without boundaries in ref. [17] a "shift" symmetry $\delta \bar{X}=-\delta \xi=\eta$ was introduced. This symmetry has been proposed also in the case of boundaries [13 where boundary conditions on $\bar{X}$ were adapted by that symmetry. In our view, although this helps to select appropriate boundary conditions, it nevertheless is not relevant for the question whether boundary conditions for $\bar{X}$ should be imposed at all.

In Appendix A a more formal derivation of the main result of the present Section for a general action with boundary condition is sketched, because this may indicate a starting point for considerations valid beyond the one-loop argument given here.

\section{Conventional sigma model}

\subsection{Background field formalism}

The action for the sigma model is usually written as

$$
S=\int_{\mathcal{M}} d^{2} z \sqrt{h}\left(\frac{1}{2} G_{\mu \nu} h^{a b} \partial_{a} X^{\mu} \partial_{b} X^{\nu}+\frac{1}{2 \sqrt{h}} \epsilon^{a b} B_{\mu \nu} \partial_{a} X^{\mu} \partial_{b} X^{\nu}+\frac{1}{2} \Phi R+T\right)
$$




$$
+\int_{\partial \mathcal{M}} d \tau\left(A_{\mu} \partial_{\tau} X^{\mu}+\hat{\Phi} k+\hat{T}\right)
$$

On the world sheet we consider Euclidean signature space coordinates $x^{a}$ and the metric $h_{a b}$ ( $R$ is the related Ricci-scalar on $\mathcal{M}, k$ the extrinsic curvature on the boundary), $X^{\mu}(x)$ are target space coordinates with metric $G_{\mu \nu}$. Further external fields are the two-form gauge field $B_{\mu \nu}$ and scalar fields $\Phi$ and $T$ on $\mathcal{M}$ and the usual gauge field $A_{\mu}$ together with the scalars $\hat{\Phi}$ and $\hat{T}$ at the boundary with tangential variable $\tau$. The string tension $\alpha^{\prime}$ has been absorbed in the external fields.

It should be emphasized that in our convention during the transition to an imaginary time coordinate we have also rotated $A_{\mu} \rightarrow i A_{\mu}$ and $B_{\mu \nu} \rightarrow i B_{\mu \nu}$. This rule of continuation has the important advantage to provide a well-posed spectral problem in Euclidean space with the same conjugation properties of all fields. Of course, that rule is not mandatory. The action with imaginary coefficients can be employed as well at the expense of introducing a more complicated conjugation operation [13]. Since hermiticity properties will play an important role in our study, we prefer to have as simple conjugation rules as possible. Actually, the fact that parity odd fields may acquire an imaginary factor in Euclidean space has been observed long ago [18] in the context of chiral theories. It is easy to see that the field $A_{\mu}$ is of odd parity from the world volume point of view. Indeed, the $A$-term in (20) can be rewritten as $\int_{M} \partial_{a}\left(\varepsilon^{a b} A_{\mu} \partial_{b} X^{\mu}\right)$. Here $A_{\mu}$ couples to the parity-odd quantity $\varepsilon^{a b}$. Whenever it is possible to compare our results to those of previous related papers [3, 目, 13], they are compatible after the replacement $A \rightarrow i A, B \rightarrow i B$.

As shown in the previous Section the expansion of (20) into a classical background solution and (geodesic) fluctuations $\xi$ allows that background field to be identified directly with the (arbitrary) mean field $\bar{X}$, when the one-loop contribution to the effective action is sought. Proceeding in the standard way [19] produces the quadratic action

$$
\begin{aligned}
S_{2}= & \frac{1}{2} \int_{\mathcal{M}} d^{2} z \sqrt{h}\left(G_{\mu \nu}(\bar{X}) h^{a b} \nabla_{a} \xi^{\mu} \nabla_{b} \xi^{\nu}-\mathcal{R}_{\mu \nu \rho \sigma}\left(\partial_{a} \bar{X}^{\mu}\right)\left(\partial^{a} \bar{X}^{\sigma}\right) \xi^{\nu} \xi^{\rho}\right. \\
& -\frac{1}{2}\left(\partial_{a} \bar{X}^{\rho}\right)\left(\partial_{b} \bar{X}^{\sigma}\right) \epsilon^{a b} \xi^{\mu} \xi^{\nu} D_{\mu} H_{\nu \rho \sigma}+\frac{1}{4}\left(\partial_{a} \bar{X}^{\nu}\right)\left(\partial^{a} \bar{X}^{\rho}\right) H_{\mu \nu \lambda} H_{\rho \sigma}^{\lambda} \xi^{\mu} \xi^{\sigma} \\
& \left.+\frac{1}{2}\left(D_{\mu} D_{\nu} \Phi(\bar{X})\right) \xi^{\mu} \xi^{\nu} R+\left(D_{\mu} D_{\nu} T(\bar{X})\right) \xi^{\mu} \xi^{\nu}\right) \\
& +\frac{1}{2} \int_{\partial \mathcal{M}} d \tau\left(\xi^{\nu} D_{\tau} \xi^{\mu}\left(B_{\mu \nu}-F_{\mu \nu}\right)+\left(\partial_{\tau} \bar{X}^{\rho}\right) D_{\mu}\left(F_{\nu \rho}-B_{\nu \rho}\right) \xi^{\mu} \xi^{\nu}\right. \\
& \left.+\left(D_{\mu} D_{\nu} \hat{\Phi}(\bar{X})\right) \xi^{\mu} \xi^{\nu} k+\left(D_{\mu} D_{\nu} \hat{T}(\bar{X})\right) \xi^{\mu} \xi^{\nu}\right) .
\end{aligned}
$$

All necessary techniques can be found in [13, 19]. 
In (21) $\mathcal{R}$ denotes the target space Riemann tensor corresponding to the target space metric $G_{\mu \nu}(\bar{X}), D_{\mu}$ is the covariant derivative for that metric,

$$
D_{a} \xi^{\mu}=\partial_{a} \xi^{\mu}+\gamma_{\nu \rho}^{\mu}\left(\partial_{a} \bar{X}^{\rho}\right) \xi^{\nu}
$$

with the target space Christoffel connection $\gamma$. The full covariant derivative

$$
\nabla_{a} \xi^{\mu}=D_{a} \xi^{\mu}+\frac{1}{2} \epsilon_{a}^{b}\left(\partial_{b} \bar{X}^{\nu}\right) H_{\nu \sigma}^{\mu} \xi^{\sigma}
$$

also depends on $H_{\mu \nu \rho}=D_{\mu} B_{\nu \rho}+D_{\nu} B_{\rho \mu}+D_{\rho} B_{\mu \nu}$. With our sign conventions $\epsilon^{N \tau}=1$.

Requiring vanishing variation of $\xi$ at $\partial \mathcal{M}$ one finds that the evaluation of the one-loop effective action should be subject to an appropriate boundary condition for $\xi$. From the first term (21), therefore, also a contribution with normal derivative $\partial_{N}=N^{a} \partial_{a}$ will emerge. Adding and subtracting a term, the appropriate boundary condition can be written as a symmetric operator

$$
\left.\mathcal{B} \xi\right|_{\partial M}=\left.\left(\nabla_{N}+\frac{1}{2}\left(\nabla_{\tau} \Gamma+\Gamma \nabla_{\tau}\right)+\mathcal{S}\right) \xi\right|_{\partial M}=0
$$

where

$$
\begin{aligned}
\Gamma_{\mu \nu} & =(B-F)_{\mu \nu}, \\
\mathcal{S}_{\mu \nu} & =\frac{1}{4}\left(\partial_{N} \bar{X}^{\rho}\right)\left[H^{\sigma}{ }_{\rho \mu}(F-B)_{\sigma \nu}+H_{\rho \nu}^{\sigma}(F-B)_{\sigma \mu}\right] \\
& -\frac{1}{2}\left(\partial_{\tau} \bar{X}^{\rho}\right)\left[D_{\mu}(F-B)_{\nu \rho}+D_{\nu}(F-B)_{\mu \rho}\right] \\
& -\left(D_{\mu} D_{\nu} \hat{\Phi}\right) k-\left(D_{\mu} D_{\nu} \hat{T}\right) .
\end{aligned}
$$

It is essential that both the normal and tangential derivatives in (24) are fully covariant. The boundary conditions (24) have a similar structure as ordinary Robin (or Neumann) boundary conditions. There are, however, some important differences which require a detailed discussion.

\subsection{Spectral geometry for boundary conditions with nor- mal and tangential derivatives}

Whereas normal derivative boundary terms have a long history, in the mathematical literature the study of the spectral geometry for differential operators with boundary conditions depending on tangential derivatives was initiated by Gilkey and Smith [20] 由. For that reason boundary conditions of that type are named

\footnotetext{
${ }^{1}$ When the present work was completed we have been informed by G.Esposito about an earlier paper on this subject [21].
} 
after these authors [22]. In the string-related literature boundary conditions with both normal and tangential derivatives are usually called generalized Neumann boundary conditions. Several first heat kernel coefficients have been calculated for such problems by McAvity and Osborn [23] and by Dowker and Kirsten [24]. Avramidi and Esposito [22, 25 27 lifted some commutativity assumptions and proved a simple criterion for strong ellipticity. Also [28 should be mentioned for a related calculation.

It is usually required that the operator $\mathcal{D}$ appearing in the quadratic form of the action should be elliptic. In the present case this means that both world volume metric and target space metric should be positive definite. On a compact manifold without boundary, ellipticity is enough to guarantee certain suitable properties of the spectrum of $\mathcal{D}$. For example, the number of non-positive modes can be at most finite. In order to ensure the same properties on manifolds with boundary one should also assume strong ellipticity of the boundary value problem [12]. Dirichlet and Neumann (Robin) boundary value problems are always strongly elliptic. For the more general boundary conditions (24) strong ellipticity is not fulfilled automatically. One should require that $|\Gamma|<1$, i.e. that all eigenvalues of $\Gamma$ lie between $-i$ and $+i$ [22,227]. For mathematical details we refer to the original literature. A simple example is given in the Appendix B. It is clear from that example that if strong ellipticity is lost the operator $\mathcal{D}$ acquires an infinite number of negative modes.

In the present context the value of $\Gamma$ for which strong ellipticity does not hold any more corresponds to critical values of the gauge field as observed in [29] Beyond that point the semi-classical approximation breaks down. This does not necessarily mean that no quantum theory exists. Perhaps the path integration over the negative modes could be performed non-perturbatively, as it has been done with zero modes leading to collective coordinates for excitations around the background provided by an instanton [32, 33].

As long as the boundary value problem is strongly elliptic $(|\Gamma|<1)$ the heat kernel expansion is well defined. There is an asymptotic series as $t \rightarrow+0$ of the form

$$
\operatorname{Tr}(\exp (-t \mathcal{D}))=\sum_{n \geq 0} t^{-m / 2+n} a_{n}(\mathcal{D})
$$

where $n=0, \frac{1}{2}, 1, \ldots$ Tr denotes the functional trace in the space of square integrable functions.

For a Laplace type operator

$$
\mathcal{D}=-\left(\nabla_{a} \nabla^{a}+E\right)
$$

and the boundary conditions (24) the heat kernel coefficient $a_{1}$ has been calcu-

\footnotetext{
${ }^{2}$ Some consequences of the existence of such a critical value for noncommutative geometry have been considered in [30,31.
} 
lated in [23]:

$$
\begin{aligned}
a_{1}= & \frac{1}{(4 \pi)^{m / 2}}\left[\int_{\mathcal{M}} \operatorname{tr}\left(E+\frac{R}{6}\right)\right. \\
& \left.+\int_{\partial \mathcal{M}} \operatorname{tr}\left(b_{0} k+b_{2} \mathcal{S}+\sigma_{1} k \Gamma^{2}\right)\right],
\end{aligned}
$$

with

$$
\begin{aligned}
& b_{2}=\frac{2}{1+\Gamma^{2}}, \\
& b_{0}+\sigma_{1} \Gamma^{2}=\frac{1}{3} .
\end{aligned}
$$

\subsection{Divergences}

In order to isolate the divergent terms we make use of the $\zeta$-function regularization [34]. The zeta function of an elliptic operator $D$ is defined as

$$
\zeta_{\mathcal{D}}(s)=\operatorname{Tr}\left(\mathcal{D}^{-s}\right) .
$$

In term of the zeta function (30) the effective action reads

$$
W=\lim _{s \rightarrow 0}\left[-\frac{1}{2 s} \zeta_{\mathcal{D}}(0)-\frac{1}{2} \zeta_{\mathcal{D}}^{\prime}(0)\right]
$$

where the prime denotes differentiation with respect to $s$.

The divergent part of the effective action follows from the well-known relation between the zeta function and the heat kernel coefficients

$$
\zeta_{\mathcal{D}}(0)=a_{1}(1, \mathcal{D}, \mathcal{B})
$$

In our case $a_{1}$ is given by (28), (29). The endomorphism $E$ can be read off from (21):

$$
\begin{gathered}
E_{\rho \nu}=\frac{1}{2} \partial_{a} \bar{X}^{\mu} \partial_{b} \bar{X}^{\sigma} \epsilon^{a b} D_{\rho} H_{\nu \mu \sigma}-\frac{1}{4} \partial_{a} \bar{X}^{\mu} \partial_{b} \bar{X}^{\mu^{\prime}} h^{a b} H_{\rho \mu}{ }^{\sigma} H_{\sigma \mu^{\prime} \nu} \\
+\mathcal{R}_{\mu \nu \rho \sigma}\left(\partial_{a} \bar{X}^{\mu}\right)\left(\partial^{a} \bar{X}^{\sigma}\right)-\frac{1}{2} R\left(D_{\rho} D_{\nu} \Phi\right)-D_{\rho} D_{\nu} T
\end{gathered}
$$

Thus the divergent part of the effective action becomes

$$
\begin{aligned}
W_{\mathrm{div}}=- & \frac{1}{8 \pi s} \int_{\mathcal{M}} d^{2} x \sqrt{h}\left[\partial_{a} \bar{X}^{\mu} \partial_{b} \bar{X}^{\rho} h^{a b}\left(-\frac{1}{4} H_{\nu \mu}{ }^{\sigma} H_{\sigma \rho \nu}+\mathcal{R}_{\mu \rho}\right)\right. \\
& \left.+\frac{1}{2} \partial_{a} \bar{X}^{\mu} \partial_{b} \bar{X}^{\sigma} \epsilon^{a b} D^{\nu} H_{\nu \mu \sigma}+R\left(\frac{d}{6}-\frac{1}{2} D^{\nu} D_{\nu} \Phi\right)-D^{\nu} D_{\nu} T\right]
\end{aligned}
$$




$$
\begin{aligned}
& -\frac{1}{8 \pi s} \int_{\partial \mathcal{M}} d \tau\left[\left(\partial_{N} \bar{X}^{\rho}\right) H_{\sigma}{ }^{\rho \mu}(F-B)^{\sigma \nu}\left(1+(F-B)^{2}\right)_{\mu \nu}^{-1}\right. \\
& -\left(\partial_{\tau} \bar{X}^{\rho}\right) 2 D^{\mu}(F-B)_{\rho}^{\nu}\left(1+(F-B)^{2}\right)_{\mu \nu}^{-1} \\
& +k\left(\frac{d}{3}-2\left(D^{\mu} D^{\nu} \hat{\Phi}\right)\left(1+(F-B)^{2}\right)_{\mu \nu}^{-1}\right) \\
& \left.-2\left(D^{\mu} D^{\nu} \hat{T}\right)\left(1+(F-B)^{2}\right)_{\mu \nu}^{-1}\right]
\end{aligned}
$$

where $d$ is dimension of the target space.

This is the most general expression ever derived, though essential parts of it have been already given in [4, 17, 13]. The most remarkable term is the one with $\partial_{N} \bar{X}$ :

$$
\left(\partial_{N} \bar{X}^{\rho}\right) H_{\sigma}{ }^{\rho \mu}(F-B)^{\sigma \nu}\left(1+(F-B)^{2}\right)_{\mu \nu}^{-1},
$$

which originates from $b_{2} S$ in (28). Such a term is absent in the bare action (20). This means that the model (20) as it stands is at least not multiplicatively renormalizable. Actually that term (35) had been noticed already before in the one-loop divergences, as calculated in [4, 7] and in the conformal anomaly [13.

Clearly one way to eliminate that disturbing term would be to assume that $\partial_{N} \bar{X}$ can be removed using the boundary conditions for the background field [4]. This amounted to assuming $\bar{X}$ to be "on-shell". As argued in Sec. 2, our generalization consists in identifying $\bar{X}$ with the mean field and therefore to be arbitrary. Already in [7] it was suggested to introduce a new coupling to restore multiplicative renormalizability. To deal with boundary divergences it was proposed in [10] to give the background fields $G_{\mu \nu}, B_{\mu \nu}$, etc. an explicit dependence on the world-volume coordinates. From the sigma model point of view this would correspond to the introduction of infinitely many new couplings which we want to avoid.

It would be natural to also consider the question, whether one can add more couplings with proper dimension and symmetries. The only possibility seems to be the volume term:

$$
\int_{\mathcal{M}} d^{2} x \sqrt{h} v_{\mu}^{a} \partial_{a} X^{\mu}
$$

Consequences of this coupling have been studied by Osborn [13]. This term would modify the connections in (22) and (23) and make the equation (34) considerably more lengthy, but would not introduce a qualitative change. Another possible candidate could be a bulk term $K_{\mu} \partial^{2} X^{\mu}$. However, by integration by parts this term can be absorbed in a redefinition of $G_{a b}$ and the $\partial_{N} X^{\mu}$ coupling.

In quantum field theory any "new" term, appearing as a counter term in the action does not necessarily imply nonrenormalizability. E.g. a regularization scheme which breaks Lorentz invariance would introduce such counterterms automatically. Actually the situation is not so different here. In principle the appearance of many possible types of new counterterms at the boundary of a 
manifold should be possible, because the invariances of the theory are broken there. We cannot totally exclude the possibility that the counterterm (35) can be removed by a field redefinition, but this seems to be very unlikely. Indeed, the fact the new coupling provides a rather beautiful mechanism for the emergence of $D$-branes (cf. [8,9] and Sec. 4.4 below) may represent a strong argument against this possibility. The final argument whether a new counter term indeed leads to a nonrenormalizable theory must come from its eventual effect on some physical observable (S-matrix element with on shell external lines (5), or, if that counterterm produces a "genuine" anomaly relative to some symmetry which is broken at the quantum level.

In our present paper we follow neither of these lines. Instead, we regard the appearance of (35) as an indication for the necessity to introduce a new coupling of $\partial_{N} X^{\mu}$ to another vector field $V_{\mu}$ at the boundary. Such a field has been proposed before [7 [11,35, 36] though our treatment of that field is different. At the moment there is no indication that the new coupling does not affect the physical content of the theory. On the contrary, it just seems to be the missing link between different string/brane models. In order to investigate its consequences in detail we consider its main features in a simplified string model in the next Section.

\section{Sigma model with $\partial_{N} X$ coupling}

\subsection{The model}

To simplify the discussion we drop all dimensional couplings from (20) and consider the action with the $V$ field present from the start

$$
\begin{aligned}
S= & \int_{\mathcal{M}} d^{2} z \sqrt{h}\left(\frac{1}{2} G_{\mu \nu} h^{a b} \partial_{a} X^{\mu} \partial_{b} X^{\nu}+\frac{1}{2 \sqrt{h}} \epsilon^{a b} B_{\mu \nu} \partial_{a} X^{\mu} \partial_{b} X^{\nu}\right) \\
& +\int_{\partial \mathcal{M}} d \tau\left(A_{\mu}(X) \partial_{\tau} X^{\mu}-V_{\mu}(X) \partial_{N} X^{\mu}\right) .
\end{aligned}
$$

The invariances of the new term can be most easily seen if it is represented as a bulk integral

$$
\int_{\mathcal{M}} d^{2} x \sqrt{h} \nabla_{R}^{a} V_{\mu} \partial_{a} X^{\mu},
$$

where $\nabla_{R}^{a}$ is the Riemannian covariant derivative on $\mathcal{M}$. Since $X^{\mu}$ is a scalar on the world sheet, the expression under the integral (38) is a scalar density. Variation of of the action (38) under the diffeomorphism $x^{a} \rightarrow x^{a}+\eta^{a}$ is therefore

\footnotetext{
${ }^{3}$ Of course, if we put the mean field $\bar{X}$ on shell, in the conventional sigma model $(20)$ the dangerous term $\partial_{N} \bar{X}$ in the one-loop effective action (34) can be traded for a term containing $\partial_{\tau} \bar{X}$ [ []. However, the corresponding divergence can resurface at higher orders of the loop expansion.
} 
given by the surface integral:

$$
\int_{\partial \mathcal{M}} d \tau \eta^{N} \nabla_{R}^{a} V_{\mu} \partial_{a} X^{\mu}
$$

This variation vanishes for $\left.\eta^{N}\right|_{\partial \mathcal{M}}=0$, i.e. for the diffeomorphisms which do not shift the boundary. In particular, the new coupling is invariant under the one-dimensional diffeomorphism transformations of the boundary, in agreement with the standard property of diffeomorphism invariant actions.

There is no explicit gradient symmetry associated with $V_{\mu}$. However, the introduction of a transformation similar to a gauge transformation could be envisaged, involving $V_{\mu}$ in the same way as it has been done with the coupling (36) (cf. eq. (4.3) of [13]). Since any symmetry of such a type will be of no consequences for our present work we will not persue this point any further here. On the other hand, an argument against the interpretation of $V_{\mu}$ as a gauge field could be that, from the point of string theory, it prepresents an off-shell degree of freedom whose physical content is still to be determined.

Proceeding as before for the general model we arrive at

$$
\begin{aligned}
S_{2} & =-\frac{1}{2} \int_{\mathcal{M}} d^{2} z \sqrt{h} \xi^{\mu}\left(\nabla^{a} \nabla_{a}+E\right)_{\mu \nu} \xi_{\nu}+\frac{1}{2} \int_{\partial \mathcal{M}} d \tau\left(-\xi^{\nu}\left(G_{\nu \mu}+2 D_{\nu} V_{\mu}\right) \nabla_{N} \xi^{\mu}\right. \\
& -\left(D_{\rho} D_{\sigma} V_{\mu}\right)\left(\partial_{N} \bar{X}^{\mu}\right) \xi^{\rho} \xi^{\sigma}+2\left(\partial_{\tau} \bar{X}^{\lambda}\right)\left(D_{\nu} V_{\mu}\right) H_{\lambda \sigma}^{\mu} \xi^{\nu} \xi^{\sigma} \\
& \left.+\xi^{\nu} D_{\tau} \xi^{\mu}\left(B_{\mu \nu}-F_{\mu \nu}\right)+\left(\partial_{\tau} \bar{X}^{\rho}\right) D_{\mu}\left(F_{\nu \rho}-B_{\nu \rho}\right) \xi^{\mu} \xi^{\nu}\right)
\end{aligned}
$$

where $E$ coincides with (33) when $\Phi=T=0$. The covariant derivative $\nabla$ is given in (23).

The natural inner product in the space of fluctuations reads:

$$
<\xi_{(1)}, \xi_{(2)}>=\int_{\mathcal{M}} d^{2} x \sqrt{h} G_{\mu \nu}(\bar{X}) \xi_{(1)}^{\mu} \xi_{(2)}^{\nu}
$$

\subsection{Boundary conditions}

To discuss the boundary conditions we now need a somewhat more general setting. The main features of (37) are reflected in the structure

$$
S=\frac{1}{2} \int_{\mathcal{M}}\left((\nabla \xi)^{2}-\xi E \xi\right)+\frac{1}{2} \int_{\partial \mathcal{M}}\left(-\xi \Lambda \nabla_{N} \xi+\xi L \xi\right),
$$

of the action, where $E$ and $\Lambda$ are the appropriate matrix-valued functions on $\mathcal{M}$ and $\partial \mathcal{M}$, respectively, and $L$ is a differential operator on the boundary $\partial \mathcal{M}$. We split $L$ into a hermitian and an antihermitian piece:

$$
L=L_{+}+L_{-}, \quad L_{ \pm}= \pm L_{ \pm}^{\dagger}
$$


Note, that $L_{+}$is fixed by the action (40) while $L_{-}$is arbitrary, since it drops out from (37) or (42). $E$ is supposed to be hermitian.

At first we consider $\Lambda=0$. In this case there is a convenient way to derive the spectral problem corresponding to the action (40). Variation of the action (40) with respect to $\xi$ leads to

$$
\delta S=-\int_{\mathcal{M}}(\delta \xi)\left(\nabla^{2}+E\right) \xi+\int_{\partial \mathcal{M}}(\delta \xi)\left(-\nabla_{N} \xi+L_{+} \xi\right) .
$$

The bulk integral in (44) contains the relevant elliptic operator $\mathcal{D}=-\left(\nabla^{2}+E\right)$, while the surface part defines the boundary conditions which can be either of Dirichlet type

$$
\left.\xi\right|_{\partial \mathcal{M}}=0
$$

(following from $\left.\delta \xi\right|_{\partial M}=0$ after absorption of an inhomogeneous mode of $\xi$ into the background), or of modified Neumann type

$$
\left.\left(-\nabla_{N} \xi+L_{+} \xi\right)\right|_{\partial M}=0 .
$$

For both cases (45) and (46) two important properties are to be noted: (i) the operator $\mathcal{D}$ is formally self-adjoint with respect to (41):

$$
<\xi_{(1)}, \mathcal{D} \xi_{(2)}>=<\mathcal{D} \xi_{(1)}, \xi_{(2)}>
$$

and (ii) after integration by parts in (40) or (42) the surface term vanishes. Due to (i) the operator $\mathcal{D}$ possesses a complete set of orthonormal eigenfunctions $\psi_{n}$ corresponding to the eigenvalues $\lambda_{n}: \psi(x)=\sum_{n} \psi_{n}(x) c_{n}$. Due to (ii) the action (40) takes the form $S=\frac{1}{2} \sum_{n} c_{n}^{2} \lambda_{n}$, and the path integral for the effective action $W$ becomes

$$
W=-\ln \left(\prod_{n} \lambda_{n}\right)^{-1 / 2}=-\ln (\operatorname{det} \mathcal{D})^{-1 / 2}
$$

where we assume a suitable regularization of the infinite product.

If $\Lambda \neq 0$ the procedure described above is not as easy to apply. The reason is that on the boundary $\delta \xi$ and $\delta \nabla_{N} \xi$ are independent. However, we can still select two relevant sets of the boundary conditions by requiring (i) and (ii) to hold. It is clear that (ii) is true for Dirichlet boundary conditions (45) and for modified Neumann conditions

$$
\left.\left(-(1+\Lambda) \nabla_{N} \xi+L \xi\right)\right|_{\partial M}=0 .
$$

To satisfy (i) we should require

$$
\left((1+\Lambda)^{-1} L\right)^{\dagger}=(1+\Lambda)^{-1} L,
$$

i.e. the operator $(1+\Lambda)^{-1} L$ should be hermitian with respect to the restriction of (41) to the boundary. The equation (50) will be used to define $L_{-}$in the decomposition (43). 
From the mathematical point of view both conditions (45) and (49) are admissible and lead to a consistent quantum field theory. From the physical point of view the conditions (49) are much superior. The first reason is that it does not seem natural for the boundary conditions to be completely independent of the boundary couplings. The second and more important one is that the Dirichlet conditions (45) are in fact contained as a special case in the generalized Neumann ones (49). This property of the boundary conditions (49) (which provides a mechanism of spontaneous creation of $D$-branes) will be discussed in sec. 4.4.

We conclude this section by comparing our boundary conditions with the ones in the existing literature. For duality reasons the coupling $V_{\mu} \partial_{N} X^{\mu}$ has been introduced in [8,9]' However, a different set of mixed boundary conditions has been proposed [9]

$$
\begin{aligned}
& f_{, A}^{\mu} G_{\mu \nu} \partial_{N} X^{\mu}+\left.(B-F)_{A B} \partial_{\tau} \eta^{B}\right|_{\partial \mathcal{M}}=0 \\
& \left.V_{\mu}\right|_{\partial \mathcal{M}}=0
\end{aligned}
$$

instead of our conditions (49). Here $\eta^{A}$ are coordinates on the $D$-brane, $f_{, A}^{\mu}$ are projectors on the $D$-brane directions. The boundary conditions (51) are fully legitimate and lead to a self-consistent sigma model. Of course, to obtain (51) the $D$-brane need be introduced from outside. In our approach it appears spontaneously in the open string sigma model (see Sec. 4.4). Also, as it is clear from Sec. 2, if one starts with the conventional open string sigma model $(V=0)$ and then turns on the $V$-field by making insertions in the propagator, one would arrive at our boundary conditions (49) without any further assumptions.

\subsection{Beta functions}

To calculate the beta functions the operator $L_{-}$in the decomposition (43), as defined by the condition (50), must be determined first. Let $(1+\Lambda)^{-1}=1+l$. Then (50) yields

$$
L_{-}=\frac{1}{2}\left(L_{+} l^{\dagger}-l L_{+}-L_{-} l^{\dagger}-l L_{-}\right)
$$

which admits an iterative solution. Let $L_{-}^{(n)}$ be a part of $L_{-}$of $n$th order in $l$ so that $L_{-}=\sum_{n=0}^{\infty} L_{-}^{(n)}$. With

$$
\begin{aligned}
& L_{-}^{(0)}=0 \\
& L_{-}^{(1)}=\frac{1}{2}\left(L_{+} l^{\dagger}-l L_{+}\right)
\end{aligned}
$$

one gets the $n$-th term

$$
L_{-}^{(n)}=-\frac{1}{2}\left(L_{-}^{(n-1)} l^{\dagger}+l L_{-}^{(n-1)}\right),
$$

\footnotetext{
${ }^{4}$ A coupling to a normal derivative at the boundary has been introduced also in order to include recoil operators of the D-brane in a description of scattering, following the Liouville approach [35] 37].
} 
and thus all finite order contributions $L_{-}^{(n)}$ are easily determined in powers of $l$ and $l^{\dagger}$, for example,

$$
\begin{aligned}
& L_{-}^{(2)}=-L_{+}\left(\frac{l^{\dagger}}{2}\right)^{2}+\left(\frac{l}{2}\right)^{2} L_{+} \\
& L_{-}^{(3)}=L_{+}\left(\frac{l^{\dagger}}{2}\right)^{3}+\left(\frac{l}{2}\right) L_{+}\left(\frac{l^{\dagger}}{2}\right)^{2}-\left(\frac{l}{2}\right)^{2} L_{+}\left(\frac{l^{\dagger}}{2}\right)-\left(\frac{l}{2}\right)^{3} L_{+} .
\end{aligned}
$$

It is easily verified that the integral

$$
L_{-}=\frac{1}{2} \int_{0}^{\infty} d a e^{-a} \exp \left(-\frac{l a}{2}\right)\left(L_{+} l^{\dagger}-l L_{+}\right) \exp \left(-\frac{l^{\dagger} a}{2}\right)
$$

summarizes $L_{-}$to all orders in $l$ and $l^{\dagger}$.

The strategy for the calculation of the heat kernel coefficient $a_{1}$ is rather simple. Given $L_{-}$one should re-write the boundary condition as

$$
\left.\left(-\nabla_{N}+(1+\Lambda)^{-1} L\right)\right|_{\partial M}=0
$$

and then transform it to the form (24) thus defining the matrices $\Gamma$ and $S . a_{1}$ then follows from $(28)$.

When $l=(1+\Lambda)^{-1}-1$ is expanded in terms of $\Lambda$, the first order contribution is $l_{\mu \nu}=-2\left(D_{\mu} V_{\nu}\right)$. Since $V$ is physically a more relevant expansion parameter than $l$, we re-expand all terms in $a_{1}$ (28) to first order of $V$. After long but otherwise elementary calculations from (40), (42), (43), (54), (31), (32) and (28) the divergent part of the effective action at $\partial \mathcal{M}$ is obtained:

$$
\begin{aligned}
W_{\operatorname{div}}[\partial \mathcal{M}] & =-\frac{1}{4 \pi s} \int_{\partial \mathcal{M}} d \tau\left(1+\Gamma_{0}^{2}\right)_{\mu \nu}^{-1}\left[( \partial _ { \tau } \overline { X } ^ { \rho } ) \left\{S_{\rho, \nu \mu}^{(\tau)}-2\left(D_{\nu} V_{\sigma}\right) H_{\rho \mu}^{\sigma}\right.\right. \\
& -2\left(\Gamma_{0, \nu \sigma}\left(D_{\sigma} V_{\lambda}\right) \Gamma_{0, \lambda \alpha}+\left(D_{\nu} V_{\sigma}\right) \Gamma_{0, \sigma \alpha}^{2}\right)\left(1+\Gamma_{0}^{2}\right)_{\alpha \beta}^{-1} S_{\rho, \beta \mu}^{(\tau)} \\
& \left.+\left(D_{\rho} D_{\nu} V_{\sigma}\right) \Gamma_{0, \sigma \mu}-2\left(D_{\nu} V_{\sigma}\right) S_{\rho, \sigma \mu}^{(\tau)}\right\} \\
& +\left(\partial_{N} \bar{X}^{\rho}\right)\left\{S_{\rho, \nu \mu}^{(N)}-2\left(D_{\nu} V_{\sigma}\right) S_{\rho, \sigma \mu}^{(N)}+\left(D_{\nu} D_{\mu} V_{\rho}\right)\right. \\
& \left.\left.-2\left(\Gamma_{0, \nu \sigma}\left(D_{\sigma} V_{\lambda}\right) \Gamma_{0, \lambda \alpha}+\left(D_{\nu} V_{\sigma}\right) \Gamma_{0, \sigma \alpha}^{2}\right)\left(1+\Gamma_{0}^{2}\right)_{\alpha \beta}^{-1} S_{\rho, \beta \mu}^{(N)}\right\}\right]
\end{aligned}
$$

The bulk terms are the same as in (34). The abbreviations

$$
\begin{aligned}
S_{\rho, \mu \nu}^{(N)} & =\frac{1}{4}\left[H_{\rho \mu}^{\sigma}(F-B)_{\sigma \nu}+\mu \leftrightarrow \nu\right], \\
S_{\rho, \mu \nu}^{(\tau)} & =-\frac{1}{2}\left[D_{\mu}(F-B)_{\nu \rho}+\mu \leftrightarrow \nu\right], \\
\Gamma_{0, \mu \nu} & =(B-F)_{\mu \nu} .
\end{aligned}
$$

have been introduced. The beta functions $\beta_{\rho}^{A}$ and $\beta_{\rho}^{V}$ to linear order in $V$ can be easily extracted from (60). It should be recalled that from all background 
couplings we had retained $A, B$ and $V$ only. The target space geometry is assumed to be flat. Therefore no distinction between upper and lower indices in (60) need to be made and the covariant derivative $D_{\mu}(22)$ coincides with the partial derivative $\partial_{\mu}$.

There is one particular case which can be analysed without additional calculations. It corresponds to constant $B$-field or $H_{\mu \nu \rho}=0$. It is clear that in this case the 0th order (in $l$ ) contribution to the beta functions are given by the expressions with $V=0$ derived in the previous section. To this order $\beta_{\mu}^{V}$ vanishes if $H_{\mu \nu \rho}=0$. Therefore, the equation $\beta_{\mu}^{V}=0$ has a solution $V=0$. This can be checked with the help of equation (60). Then the beta function $\beta_{\mu}^{A}$ is given by its old expression and is proportional to variation of the Born-Infeld action with respect to $A$. Hence, for constant $B$ field we reproduce the Born-Infeld action as one of the possible solutions. There could be also non-perturbative ones when $V$ is not small. Also, at least for small $V$, the expansion in $V$ or $l$ corresponds to an expansion in derivatives of the $B$ field.

\subsection{Spontaneous creation of $D$-branes}

The boundary conditions (49)

$$
\left.\left[\left(\delta_{\mu \nu}+2 D_{\mu} V_{\nu}\right) \nabla_{N}+L_{\mu \nu}\right] \xi^{\nu}\right|_{\partial \mathcal{M}}=0
$$

have intriguing qualitative features. Let us consider the simplest case when $D_{\mu} V_{\nu}=D_{\nu} V_{\mu},\left[D V, \Gamma_{0}\right]=0$, where $\Gamma_{0}=B-F$. If we rewrite (62) in the form (59) the operator $\Gamma$ becomes

$$
\Gamma=(1+2 D V)^{-1} \Gamma_{0}
$$

When $(1+2 D V)^{-1}$ is sufficiently large so that the absolute value of some of the eigenvalues of $\Gamma$ becomes larger than 1, strong ellipticity is lost, and, as discussed in sec. 3.2, we enter a non-perturbative strong coupling regime. There an infinite number of negative modes appears which correspond to tachyonic modes in the Minkowski signature theory.

On the other hand, when the matrix $(1+2 D V)$ becomes degenerate, so that $(1+2 D V) \xi^{[0]}=0$ for some $\xi^{[0]} \neq 0$, (62) implies

$$
\left.L \xi^{[0]}\right|_{\partial \mathcal{M}}=0
$$

or, since for generic values of the other background couplings $A, B$, etc., $L$ is non-degenerate -this should be true at least on the zero-measure subset where $(1+2 D V)$ is degenerate-

$$
\left.\xi^{[0]}\right|_{\partial \mathcal{M}}=0 .
$$

This clearly indicates the formation of a $D$-brane. The co-dimensionality of that $D$-brane is equal to number of zero eigenvalues of $(1+2 D V)$. Note that at this 
point strong ellipticity is recovered again, but with a different type of boundary conditions. We conclude that the model with $V$-coupling describes both open strings and $D$-branes depending on the value of the derivatives of the field $V$.

Multiple roots of $(1+2 D V)$ correspond to multiple $D$-branes sitting on top of each other. Such configurations should produce non-abelian Chan-Paton factors [38].

With some restrictions on the form of the background couplings it is possible to calculate the $\beta$-functions without expanding in power series of $V$. In this way a clearer picture of $D$-brane formation can be obtained. We postpone this task for a future publication.

The present mechanism of $D$-brane formation should be compared with the one proposed and studied in the previous literature [39 45]. The mechanism proposed there in our framework corresponds to a dominating $L$ in the boundary conditions (62) (with $V=0$ ), so that this condition (62) becomes approximately Dirichlet and an exact Dirichlet boundary condition could be achieved for infinite $L$ only. In our case Dirichlet boundary conditions appear for finite background couplings due to the vanishing of the coefficient in front of the normal derivative. Of course, in the framework of our approach also a combination of the two mechanisms could be envisaged.

\section{Conclusions}

We have devoted much space (Sec. 2 and Appendix A) to the consideration of the effective action in the presence of boundaries. It seems that a completely satisfactory construction of such an object is still missing in the literature. We do not pretend to have obtained the ultimate solution as well. However, we were able to extract two important lessons from our considerations. First, we have seen that to be able to reproduce reliably all divergences of the 1-p-i diagrams one should not impose any restriction on the mean field. In particular, the latter should not satisfy any boundary conditions. Second, we have shown how the boundary conditions are changed due to "perturbative" insertions of boundary interactions in the propagator. In rederiving the divergent part of the effective action for conventional open string sigma model some mathematical issues like strong ellipticity of the boundary value problem have been clarified. Also, we were able to obtain exact results for slightly more general couplings than the ones exhibited in previous calculations. We also reconsidered the approach of [4] where the mean field $\bar{X}$ was taken on-shell. While this worked perfectly for the renormalization of the one-loop physical amplitudes, to analyse multiplicative renormalizability of the full theory we suggest to to extend the method of [4] to an off-shell mean field $\bar{X}$ and so that classical boundary conditions are no longer relevant for it. Our analysis strongly suggests the introduction of a new coupling $V_{\mu} \partial_{N} X^{\mu}$ at the boundary. In fact, such a step has been proposed long ago [7]. 
Therefore, the sigma model with that new coupling has been studied. After formulating the boundary value problem, the $\beta$-functions to the leading order in $V$ were calculated. For constant $B$-field we found that one of the beta functions, $\beta^{V}$, vanishes for $V=0$ while the other, $\beta^{A}$, then reproduces the variation of the BornInfeld action. The perturbative expansion in terms of small $V$ generates derivative corrections to that action?. It would be interesting to compare the derivative corrections obtained by the beta function method with the ones following from the Seiberg-Witten transformation [50]. Of course, this would require a considerable improvement of our understanding of non-commutative geometry in the presence of a non-constant $B$-field (see [51] for some recent results and further references).

To us the most exciting feature of the new coupling is that a natural mechanism of spontaneous $D$-brane creation is implied. As the matrix $\left(\delta_{\mu \nu}+2 D_{\mu} V_{\nu}\right)$ becomes degenerate the boundary conditions (62) are transformed to mixed Dirichlet-Neumann ones, thus describing a $D$-brane with co-dimensionality equal to the number of zero eigenvalues of that matrix.

In our view, the $V$-coupling deserves further investigation. In particular, one should clarify the duality properties of the modified sigma model. Even though our model (37) for the string with boundary terms looks very similar to the one proposed in [8, 9], the boundary conditions are different, so that $T$ duality transformations may require modification. Of course, another interesting generalization of our results would be the inclusion of supersymmetry.

\section{Acknowledgements}

This work has been supported by the FWF (Fonds zur Förderung der wissenschaftlichen Forschung) project P-12.815-TPH, the Alexander von Humboldt Foundation and the DFG, grant Bo 1112/11-1. One of the authors (DV) is grateful to the Erwin Schrödinger Insitute for Mathematical Physics for warm hospitality. We are grateful to A. Andrianov, I.Avramidi, I.Bandos, G.Esposito, M.Kreuzer and H.Dorn for discussions and thank the referee for constructive remarks.

\section{Appendix A: One-loop effective action and clas- sical background field (a formal argument)}

1-p-i-vertices may be extracted from the effective action $W\left(\bar{\phi}_{i}\right)$ where $\bar{\phi}$ is the "mean field",

\footnotetext{
${ }^{5}$ Derivative corrections to the Born-Infeld action for vanishing or constant $B$-field as power series in the $F_{\mu \nu}$ generated by higher loops were calculated in [46, 477. Recently the emergence of such corrections in non-commutative geometry was also considered [48, 49].
} 


$$
\bar{\phi}_{i}=\frac{\delta Z_{c}}{\delta j_{i}},
$$

related to the generating functional $Z(j)$ of Green-functions $Z=\exp i Z_{c}$ by differentiation with respect to sources $j_{i}$ in the latter. We use deWitt's notation with the index $i$ describing the space-time variable as well as (eventual) indices of internal symmetries and in this Appendix we work in Minkowski space. Actually the action $S=S^{\mathcal{M}}+S^{\partial \mathcal{M}}$ in $Z$ may also contain a source term at the boundary.

The relation of $\bar{\phi}_{i}$ to a generic classical solution $\underline{\phi}_{i}$, around which quantum fluctuations $\varphi_{i}$ may occur, is not immediate, especially when the quantum system — and hence $\underline{\phi}_{i}$ - obeys certain boundary conditions

$$
F_{\alpha}(\phi)=0
$$

which must be imposed. A dependence on external fields is not indicated explicitly. For our present purposes it is sufficient to consider theories without gauge invariance, where $Z(j)$ may be simply generalized as

$$
Z(j, k)=\int(d \phi)(d \lambda) C e^{i\left(\frac{S}{\hbar}+j_{i} \phi_{i}+\lambda_{\alpha} F_{\alpha}(\phi)+\lambda_{\alpha} k_{\alpha}\right)}
$$

to include (67) by means of a Lagrange multiplier field $\lambda_{\alpha}$ (Greek indices indicate that their space-time variable and related integrals refer to the boundary $\partial \mathcal{M})$. It is then convenient to introduce a source $k_{\alpha}$ for that field too (thus restricted to $\partial \mathcal{M}$ as well) . The factor $C$ should be determined by the requirement that the actual path integral should involve only the modes obeying the boundary conditions. Its derivation in the fully general case would imply the development of a Hamiltonian formalism, including boundary constraints, adapted to the present situation which does not seem to be available. Certain similarities to the FaddeevPopov determinant can be expected. A formulation like (68) has the advantage that the boundary conditions are completely integrated into the formalism and not imposed "from the outside".

The expansion $\phi_{i}=\phi_{i}+\varphi_{i}$ around a "background" solution $\underline{\phi_{i}}$ in (3) requires the "saddle point" condition for the linear term in $\varphi_{i}$ and $\lambda_{\alpha}$

$$
\begin{aligned}
& \frac{1}{\hbar} \frac{\delta S}{\delta \underline{\phi_{i}}}+j_{i}=0, \\
& \left.F_{\alpha} \underline{\phi}\right)+k_{\alpha}=0
\end{aligned}
$$

which only for vanishing sources $j_{i}, k_{\alpha}$ implies that the solutions $\phi_{i}$ are the ones of the classical e.o.m.-s, subjected to the boundary conditions (67). Retaining in (68) only quadratic terms in $\varphi_{A}=\left(\varphi_{i}, \lambda_{\alpha}\right)$ after some simple formal manipulations yields for the mean field (66) and an analogous one related to $k_{\alpha}$

\footnotetext{
${ }^{6}$ In order to be able to reproduce the Green functions for fields at $\partial \mathcal{M}, j_{i}$ may contain $\delta$-function contributions.
} 


$$
\begin{aligned}
& \bar{\phi}_{i}=\underline{\phi_{i}}(j, k)+\mathcal{O}(\hbar), \\
& \bar{\lambda}_{\alpha}=\mathcal{O}(\hbar) \text {. }
\end{aligned}
$$

Thus to leading order, (one loop in the effective action) the mean field is identical to $\phi_{i}(j, k)$, but as arbitrary as the dependence of the latter on the sources $j$ and $k$. Therefore, if the evaluation of the path integral in $\varphi$ around $\phi$ is performed, the simple replacement (71) yields the contribution to the effective action.

In contrast to the argument of Section 2, the result (71) is found to hold for a generic action $S$. However, it should be noted that the presence of a finite boundary $\partial \mathcal{M}$ really forbids the application of the usual rules of functional derivation, e.g. in the expansion of (67). Naive application in the case of a normal derivative contained in the boundary would lead to a surface term which rides on top of the boundary again and thus produces $\delta(0)$. Therefore, such terms must be dropped explicitly. Whether this can be made into a general consistent prescription must be the subject of a more profound analysis.

\section{Appendix B: Strong ellipticity (an example)}

For the Laplace operator

$$
\Delta=-\partial_{1}^{2}-\partial_{2}^{2}
$$

on a flat manifold $\mathcal{M}=[0,1] \times S^{1}$ with the boundary conditions

$$
\left.\partial_{1} \phi\right|_{x^{1}=0}=0,\left.\quad\left(\partial_{1}+i \alpha \partial_{2}\right) \phi\right|_{x^{1}=1}=0
$$

there are two sets of eigenmodes. The first one is:

$$
\phi_{k_{1} k_{2}}=\exp \left(i k_{2} x^{2}\right) \cos \left(k_{1} x^{1}\right)
$$

These modes satisfy (74) at $x^{1}=0$. The condition at $x^{1}=1$ should be used to define discrete values of $k_{1}$. $k_{2}$ is quantized to assure periodicity in the $x^{2}$ coordinate. Obviously, the eigenvalues $k_{1}^{2}+k_{2}^{2}$ of $\Delta$ are always non-negative. The second set of the eigenfunctions

$$
\bar{\phi}_{k_{1} k_{2}}=\exp \left(i k_{2} x^{2}\right) \cosh \left(k_{1} x^{1}\right)
$$

again satisfies (74) at $x^{1}=0$. At $x^{1}=1$ that condition yields

$$
k_{1} \tanh \left(k_{1}\right)=\alpha k_{2} .
$$

For positive $\alpha k_{2}$ (77) always has two solutions. For sufficiently large $\left|k_{2}\right|$ they are

$$
k_{1} \approx \pm \alpha k_{2}
$$


and the corrections are exponentially small. From (78) the eigenvalues $-k_{1}^{2}+k_{2}^{2}$ of $\Delta$ are now positive if $|\alpha|<1$ and negative if $|\alpha|>1$. We conclude that if strong ellipticity of the boundary value problem is lost $(|\alpha|>1)$ the operator $\Delta$ shows infinitely many negative modes.

\section{References}

[1] E.S. Fradkin and A.A. Tseytlin, Phys. Lett. 163 B (1985) 123.

[2] A.A. Tseytlin, Nucl. Phys. B 276 (1986) 391.

[3] A. Abouelsaood, C.G. Callan, C.R. Nappi and S.A. Yost, Nucl. Phys. B 280 [FS 18] (1987) 599.

[4] C.G. Callan, C. Lovelace, C.R. Nappi and S.A. Yost, Nucl. Phys. B 308 (1988) 221.

[5] C.G. Callan, D. Friedan, E.J. Martinec and M.J. Perry, Nucl. Phys. B 262 (1985) 593.

[6] A.A. Tseytlin, Born-Infeld action, supersymmetry and string theory, hepth/9908105, to appear in the Yuri Golfand memorial volume, ed. M. Shifman, (World Scientific, 2000).

[7] H. Dorn and H.-J. Otto, Z. Phys. C32 (1986) 599.

[8] J. Dai, R.G. Leigh, J. Polchinski, Mod. Phys. Lett. A 4 (1989) 2073.

[9] R.G. Leigh, Mod. Phys. Lett. A 4 (1989) 2767.

[10] K. Behrndt and H. Dorn, Int. J. Mod. Phys. A7 (1992) 1375.

[11] H. Dorn and H.-J. Otto, Phys. Lett. B 341 (1996) 81.

[12] P.B. Gilkey, Invariance Theory, the Heat Equation, and the Atiyah-Singer Index Theorem, (CRC Press, Boca Raton, 1994).

[13] H. Osborn, Nucl. Phys. B 363 (1991) 486.

[14] D.M. McAvity and H. Osborn, Nucl. Phys. B 394 (1993) 728.

[15] K. Symanzik, Nucl. Phys. B 190 [FS3] (1981) 1.

[16] C. Wiesendanger and A. Wipf, Ann. Phys. 233 (1994) 125.

[17] P.S. Howe, G. Papadopoulos and K.S. Stelle, Nucl. Phys. B 296 (1988) 26.

[18] A.A. Andrianov and L. Bonora, Nucl. Phys. B 233 (1984) 232; 247. 
[19] E. Braaten, T.L. Curtright and C.K. Zachos, Nucl. Phys. B 260 (1985) 630.

[20] P.B. Gilkey and L. Smith, The twisted index problem for manifolds with boundary, J. Diff. Geom. 15 (1983) 393.

[21] G. Grubb, Annali della Scuola Normale Superiore di Pisa 1 (1974) 1.

[22] I. Avramidi and G. Esposito, Trends in Mathematical Physics: Proceedings. Edited by V. Alexiades and G. Siopsis. Cambridge, Mass., International Press, 1999. (AMS/IP Studies in Advanced Mathematics, v. 13). pp. 15-34.

[23] D.M. McAvity and H. Osborn, Class. Quantum Grav. 8 (1991) 1445.

[24] J.S. Dowker and K. Kirsten, Class. Quantum Grav. 14 (1997) L169; 16 (1999) 1917.

[25] I. Avramidi and G. Esposito, Class. Quantum Grav. 15 (1998) 1141.

[26] I. Avramidi and G. Esposito, Class. Quantum Grav. 15 (1998) 281.

[27] I.G. Avramidi and G. Esposito, Commun. Math. Phys. 200 (1999) 495.

[28] E. Elizalde and D.V. Vassilevich, Class. Quantum Grav. 16 (1999) 813.

[29] V.V. Nesterenko, Int. J. Mod. Phys. A 10 (1989) 2627.

[30] N. Seiberg, L. Susskind and N. Toumbas, Strings in Background Electric Field, Space/Time Noncommutativity and a New Noncritical String Theory, hep-th/0005040.

[31] I. Klebanov and J. Maldacena, $1+1$ dimensional NCOS and its $U(N)$ gauge theory dual, hep-th/0006085.

[32] G. 't Hooft, Phys. Rev. D 14 (1976) 3432.

[33] H. Osborn, Ann. Phys. 135 (1981) 373.

[34] J.S. Dowker and R. Critchley, Phys. Rev. D 13 (1976) 3224; S.W. Hawking, Commun. Math. Phys. 55 (1977) 133.

[35] J. Ellis, N.E. Mavromatos and D.V. Nanopoulos, Int. J. Mod. Phys. A 12 (1997) 2639.

[36] N.E. Mavromatos and R.J. Szabo, Phys. Rev. D 59 (1999) 104018.

[37] J. Ellis, N.E. Mavromatos and D.V. Nanopoulos, A microscopic Liouville arrow of time, hep-th/9805120; Dinamical formation of horizons in recoiling $D$ branes, hep-th/0006004. 
[38] J. Polchinski, Phys. Rev. Lett. 75 (1995) 4724.

[39] A. Sen, Int. J. Mod. Phys. A 14 (1999) 4061.

[40] J.A. Harvey and P. Kraus, JHEP 0004 (2000) 012.

[41] J.A. Harvey, D. Kutasov and E.J. Martinec, On the relevance of tachyons, hep-th/0003101.

[42] J. Majumder and A. Sen, Vortex pair creation on brane-antibrane pair via marginal deformation, hep-th/0003124.

[43] R. de Mello Koch, A. Jevicki, M. Mihailescu and R. Tatar, Lumps and pbranes in open string theory, hep-th/0003031.

[44] M. Moeller, A. Sen and B. Zwiebach, D-branes as tachyons lumps in string field theory, hep-th/0005036.

[45] T.Z. Husain and M. Zabzine, Bosonic open strings in a tachyonic background fields, hep-th/0005202.

[46] O.D. Andreev and A.A. Tseytlin, Nulc. Phys. B 311 (1988) 205.

[47] O.D. Andreev and A.A. Tseytlin, Mod. Phys. Lett. A 3 (1988) 1349.

[48] Y. Okawa, Nucl. Phys. B 566 (2000) 348.

[49] L. Cornalba, Corrections to the abelian Born-Infeld action arising from noncommutative geometry, hep-th/9912293.

[50] N. Seiberg and E. Witten, JHEP 9909 (1999) 032.

[51] P.-M. Ho and Y.-T. Yeh, Non-commutative D-brane in non-constant NS-NS $B$-field, hep-th/0005159. 